\title{
Evaluation of Nurse's Knowledge in Management of Premature Baby in Neonatal Units
}

\author{
Sajjad S. Issa", Kadim Jawad AL Madwah, Hajer Salim Al Mosawi \\ The Basics of Nursing, Basrah Nursing College, Basrah University, Basrah, Iraq \\ *Corresponding author: Sajjad.issa@uobasrah.edu.iq
}

\begin{abstract}
Every year, an estimated 15 million babies are born preterm (before 37 completed weeks of gestation), and this number is rising. Preterm birth complications are the leading cause of death among children under 5 years of age, responsible for nearly 1 million deaths. Our aims were to evaluate of nurse's knowledge in management of premature baby in neonatal units (PBU). To find out relationship between socio demographic variables and nurses knowledge. To determine the relation between the experience and level of knowledge. A selected sample study carried out in four general hospital in Basra-Iraq: Basra general hospital, Almoani general hospital, Qurna general hospital and Basra hospital for Maternity and childhood. A sample of 40 nurses was included and interviewed for the purpose of the study . The enquiry forma consist of 2 part, one for the demographic data, the second part concern with evaluation of the nursing intervention for management of premature babies units, which was done by the resident doctors in these units. The second part consist of 14 items represent the roles of nurses in the PBU. Likert Scale has been used for the evaluation scores. The data has been fed in SPSS program for statistical purposes. The study Showed that $90 \%$ of the nurses was females and $10 \%$ was males, $32 \%$ were at age interval of 20 to 29 years and $50 \%$ were between 30 to 49 years. $15 \%$ were at 50 to 59 years.57.5 \% of the nurses are graduated from secondary school, $32.5 \%$ are graduated from health institute, and $7.5 \%$ from intermediate school and $2.5 \%$ are from college. $30 \%$ of the nurses were having 1 to 5 years of experience in the PBU, $25 \%$ were having 11 to 15 years, $12.5 \%$ having 6 to 10 years, $5 \%$ having 21 years, $2.5 \%$ having less than one years. Only $7.5 \%$ had a participation in training session. The mean evaluation score is $73.9 \%$, the minimum score is $57 \%$, and the maximum score is $92 \%$. At Basra, hospital for Maternity and childhood had the highest score $77 \%$, and followed by Amwani general hospital $76.56 \%$, then Quran general hospital $75 \%$, and lastly Basra general hospital $66 \%$. There is significant correlation between the scores that the nurses got and the years of their experiences.
\end{abstract}

Keywords: Evaluation of Nurse's, Knowledge in Management, Premature Baby.

Cite This Article: Sajjad S. Issa, Kadim Jawad AL Madwah, and Hajer Salim Al Mosawi, "Evaluation of Nurse's Knowledge in Management of Premature Baby in Neonatal Units." American Journal of Nursing Research, vol. 6, no. 5 (2018): 291-295. doi: 10.12691/ajnr-6-5-10.

\section{Introduction}

Every year, an estimated 15 million babies are born preterm (before 37 completed weeks of gestation), and this number is rising. Preterm birth complications are the leading cause of death among children under 5 years of age, responsible for nearly 1 million deaths. In 2013. Three-quarters of them could be saved with current, cost-effective interventions. Across countries, the rate of preterm birth ranges from $5 \%$ to $18 \%$ of babies born. [8] A Premature birth gives the neonate less time to develop in the womb. Although, premature infants especially those born earliest, often have complicated medical problems [1], meanwhile not all premature experiences complications, these depending on how early a neonate is born, where's extremely preterm born at less than 25 weeks of pregnancy. Furthermore, very preterm born at less than 32 weeks of pregnancy and late preterm born between 34 and 36 weeks of pregnancy. Generally, the earlier a neonate is born, the higher the risk of complications physiological and psychological. [4] Due to some complications associated prematurity, these infants need to hospitalize in Neonatal Intensive Care Unit (NICU) [1]. In recent years, factors such as assisted reproductive technology, old age pregnancy, advanced ante/postnatal diagnosis and treatments cause a rise in preterm birth rate [2]. The rate of preterm birth in 2005 was reported 12.9 million births (9.6\%) worldwide and about 11 million (85\%) in Africa and Asia [3]. Some newborns require observation and care that is beyond the scope of a normal newborn nursery, these are called high-risk neonates [2]. High risk neonate can be defined as a newborn, regardless of gestational age or birth weight, which has a greater-than-average chance of morbidity or mortality, requiring early intervention that should be delivered at neonatal intensive care unit [3]. It provides care to full spectrum of newborns ranging from extremely premature infants, to high risk and critically ill babies, to 
less critically ill babies who are recovering and maturing with increased emphasis is being placed on the need for standards of care, as well as mechanisms which address the barriers to provision and use of quality care [4]. The first step in improving quality of nursing care is an articulation of standards of care that provide a mean for determining quality of care as well as accountability of the nurses [5]. A standard is defined as a professionally agreed level of performance, it provides the required knowledge and skills that can be used to orient new staff and to guide nurses in clinical practice [6]. Nurses are the key elements in critical care. They are required to keep pace with the rapid changes in health care, and provide quality of patient's care in a cost-effective manner [7] Healthcare institutions have varying entry-level requirements for neonatal nurses. Neonatal nurses are registered nurses (RNs), and therefore must have an Associate of Science in Nursing (ASN) or Bachelor of Science in Nursing (BSN) degree. Some countries or institutions may also require midwifery qualification. Some institutions may accept newly graduated RNs having passed the NCLEX exam; others may require additional experience working in adult-health or medical/surgical nursing. [5] Some countries offer postgraduate degrees in neonatal nursing, such as the Master of Science in Nursing (MSN) and various doctorates. A nurse practitioner may be required to hold a postgraduate degree. [4] The National Association of Neonatal Nurses recommends two years' experience working in a NICU before taking graduate classes. [5] There are no mandated requirements to becoming an RN in a NICU, although neonatal nurses must have certification as a neonatal resuscitation provider. Some units prefer new graduates who do not have experience in other units, so they may be trained in the specialty exclusively, while others prefer nurses with more experience already under their belt. Intensive-care nurses endure intensive and clinical orientation, in addition to their general nursing knowledge, to provide highly specialized care for critical patients. Their competencies include the administration of high-risk medications, management of high acuity patients requiring ventilator support, surgical care, resuscitation, procedures, as well as chronic-care management or lower acuity cares associated with premature infants such as feeding intolerance. [6] The Neonatal Intensive Care Unit (NICU) constitutes a therapeutic environment appropriate for treatment of the newborn (NB) in a serious condition. The fragility of these NBs, the increasing implementation of high-risk procedures and the low tolerance to medication errors are some of the concerns of nursing professionals working in the NICU [22]. The constant interaction with the family members of newborns requires training of the health team to offer support to them in this moment of weakness. Furthermore, the nurse is responsible for implementing a care that valorizes the physical, mental and social development of the NB [23].

Incubator: An incubator is an apparatus used to maintain environmental conditions suitable for a neonate (newborn baby). It is used in preterm births or for some ill full-term babies. [9]

Neonatal care: Care for infants with corrected gestational age greater than 34 weeks or weight greater than $1800 \mathrm{~g}$ who have mild illness expected to resolve quickly or who are convalescing after intensive care [9].

Neonatal Units: Also known as 'Special Care Baby Units' (SCBU). These look after babies who need more care than healthy newborns but are relatively stable and mature. SCBU might provide tube-feeding, oxygen therapy, antibiotics to treat infection and phototherapy for jaundice. [9].

\section{Objectives of the Study}

1. To Evaluation of nurse's knowledge in management of premature baby in neonatal units

2. To find out relationship between socio demographic variables (age, relationship, and other), and nurses knowledge in management of premature baby in neonatal units.

3-To determine the relation between the experience and level of knowledge

\section{Methodology}

\subsection{Design of the Study}

A selected sample study designed to evaluate nurses' knowledge in management of premature babies in neonatal unit in Basra hospitals the period of data collection from December 2015 to Feb. 2016.

\subsection{Setting of the Study}

The study was carried out in four general hospital in Basra

1-Basra general hospital2-Almwani general hospital3Qurna general hospital

4- Basra hospital for Maternity and childhood

\subsection{The Sample of the Study}

A sample of 40 nurses was included and were interviewed for the purpose of the study.

\subsection{The Tools}

The enquiry forma consist of 2 part, one for the demographic data, the second part concern with evaluation of the nursing intervention for management of premature babies units, which was done by the resident doctors in these units. (Appendix 1) the second part consist of 14 items represent the roles of nurses in the PBU, with 3 answer against each role represent their abilities to perform such role and give each answer a score, these are: NEVER (score zero), SOMETIME (score one), ALWAYS (score two), then we found the TOTAL scores for every nurse, and represent the scores in percentages .

\subsection{Statistical Analysis}

The Statistical Package for Social Science (SPSS), Version16 was utilized for the purpose of statistical 
analysis of the data. The statistical measures was in form of means, frequencies, percentages, and Pearson correlation. Likert Scale has been used for the evaluation scores.

\section{Result}

Forty nurses was included in our study sample, they were working in the premature babies units in four different hospitals at Basra Governorate. They were evaluated concerning nursing interventions for management of premature babies in premature babies units.

Table 1. Distribution of the studied sample according to gender

\begin{tabular}{|l|c|c|}
\hline Gender & Frequency & Percentage \\
\hline Females & 36 & 90 \\
\hline Males & 4 & 10 \\
\hline Total & 40 & 100 \\
\hline
\end{tabular}

This table showed the frequency distribution of the sample according to gender, and it showed that $90 \%$ was females and $10 \%$ was males

Table 2. Distribution of the studied sample according to ages

\begin{tabular}{|l|c|c|}
\hline Age intervals in years & Frequency & Percentages \\
\hline $20-29$ & 13 & 32.5 \\
\hline $30-39$ & 10 & 25 \\
\hline $40-49$ & 10 & 25 \\
\hline $50-59$ & 6 & 15 \\
\hline 60 and above & 1 & 2.5 \\
\hline Total & 40 & 100 \\
\hline
\end{tabular}

This table showed that about $32 \%$ were at age interval of 20 to 29 years and $50 \%$ were between 30 to 49 years. $15 \%$ were at 50 to 59 years.

Table 3. Distribution of the studied sample according to level of education

\begin{tabular}{|l|c|c|}
\hline Levels of education & Frequency & Percentages \\
\hline Intermediate school & 3 & 7.5 \\
\hline Secondary school & 23 & 57.5 \\
\hline Health institute & 13 & 32.5 \\
\hline College graduates & 1 & 2.5 \\
\hline Total & 40 & 100 \\
\hline
\end{tabular}

This table showed the distribution of the nurses according to the level of education. $57.5 \%$ of the nurses are graduated from secondary school, $32.5 \%$ are graduated from health institute, and $7.5 \%$ from intermediate school and $2.5 \%$ are from college. That is mean most of nurses are Secondary school graduated nurses.

Table 4. Distribution of the studied sample according to marital status

\begin{tabular}{|l|c|c|}
\hline Marital status & Frequency & Percentage \\
\hline Single & 10 & 25 \\
\hline Married & 30 & 75 \\
\hline Total & 40 & 100 \\
\hline
\end{tabular}

This table showed that $75 \%$ of the nurses was married while $25 \%$ was single.

Table 5. Distribution of the studied sample according to years of experience

\begin{tabular}{|l|c|c|}
\hline years of experience & Frequency & Percentages \\
\hline Less than one year & 3 & 2.5 \\
\hline $1-5$ & 12 & 30 \\
\hline $6-10$ & 5 & 12.5 \\
\hline $11-15$ & 10 & 25 \\
\hline $16-20$ & 8 & 20 \\
\hline 21 and more & 5 & 5 \\
\hline Total & 40 & 100 \\
\hline
\end{tabular}

This table showed the years of experience, in which $30 \%$ of the nurses were having 1 to 5 years of experience in the PBU, $25 \%$ were having 11 to 15 years, $12.5 \%$ having 6 to 10 years, $5 \%$ having 21 years, $2.5 \%$ having less than one years.

Table 6. Distribution of the studied sample according to participation in training

\begin{tabular}{|l|c|c|}
\hline participation in training & Frequency & Percentage \\
\hline YES & 37 & 92.5 \\
\hline NO & 3 & 7.5 \\
\hline Total & 40 & 100 \\
\hline
\end{tabular}

This table showed that only $7.5 \%$ had a participation in training session while.

The percentage of non-participation in training session were92.5\%.

Table 7. Distribution of the studied samples according to hospitals

\begin{tabular}{|l|c|c|}
\hline Hospital name & Frequencies & Percentages \\
\hline Almawani general hospital & 9 & 22.5 \\
\hline Basra general hospital & 10 & 25 \\
\hline Maternity and childhood hospital & 11 & 27.5 \\
\hline Alqurna general hospital & 10 & 25 \\
\hline Total & 40 & 100 \\
\hline
\end{tabular}

This table show the distribution of nurses according to the hospitals where they were worked, where there were little differences.

Table 8. The mean scores of the sample

\begin{tabular}{|l|c|}
\hline Scores & Value \\
\hline Mean & 75.9 \\
\hline Minimum & 57 \\
\hline Maximum & 92 \\
\hline
\end{tabular}

The table showed the values of mean, minimum and maximum scores that the nurses got through their evaluation by resident doctors in the premature unit. The mean score is $73.9 \%$, the minimum is $57 \%$, and the maximum is $92 \%$. That is mean most nurses are doing well in the PBU. 
Table 9. Distribution of the sample according to hospital and scores

\begin{tabular}{|l|c|c|}
\hline Hospital name & Frequencies & Mean of scores \\
\hline Almawani general hospital & 9 & 76.56 \\
\hline Basra general hospital & 10 & 66 \\
\hline Maternity and childhood hospital & 11 & 77 \\
\hline Alqurna general hospital & 10 & 75 \\
\hline Total & 40 & 73.9 \\
\hline
\end{tabular}

This table showed that the nurses in PBU at Basra hospital for Maternity and childhood had the highest score $77 \%$, and followed by Amani general hospital $76.56 \%$, then Quran general hospital $75 \%$, and lastly Basra general hospital $66 \%$ which represent the lowest scores.

In addition, the table showed that the mean of total scores for all nurses was $73.9 \%$.

Table 10. Distribution of the sample according to score intervals

\begin{tabular}{|l|c|c|c|}
\hline No. & Score intervals & Frequencies & Percentages \\
\hline 1 & $51-60$ & 4 & 10 \\
\hline 2 & $61-70$ & 11 & 27.5 \\
\hline 3 & $71-80$ & 13 & 32.5 \\
\hline 4 & $81-90$ & 11 & 27.5 \\
\hline 5 & 91 and above & 1 & 2.5 \\
\hline Total & & 40 & 100 \\
\hline
\end{tabular}

This table showed the distribution of the sample according to scores intervals, $10 \%$ in the first group, $27.5 \%$ in the second and fourth group, 32.5 in the third group and 2.5 in the last group.

Table 11. Correlation of scores versus years of experiences and levels of education

\begin{tabular}{|l|c|c|c|}
\hline Spearman's correlation & Scores & Experience & Education \\
\hline Correlation coefficient & 1 & 0.315 & 0.241 \\
\hline Sig(2- tailed ) & & 0.045 & 0.134 \\
\hline N & 40 & 40 & 40 \\
\hline
\end{tabular}

The table showed that there is significant correlation between the scores that the nurses got and the years of their experiences, while there is no significant correlation between the scores that the nurses got and the level of education.

\section{Discussion}

Our study was designed to evaluate the nurses knowledge about nursing intervention in management of premature baby in neonatal units, two forma was used for the evaluation, first forma represent the demographic features for the studied nurses, the second forma consist of fourteen items represents the role of nurses in the PBU, was given to the resident doctors in the units to do the evaluation. In our study we find that , the nursing staff in PBU was mostly female in gender (90\%), which accepted because nurses might do some jobs of the mothers in such unit where male might had less abilities to do such job . $50 \%$ of the staff was 30 to 50 years old that is mean the staff in PBU had worked many years in the pediatrics wards and got a good knowledge and experience then they were shifted to PBU, to make the staff of PBU more perfect. regarding scoring we found that all nurses had passed and succeed and because they had done their jobs in right way, and they are skilled enough and able to do their duets, in our study we found that the nurses in the Nurses in Basra hospital for Maternity and childhood and Amani general hospital did their jobs better than the other hospitals where they got higher scores. also our study showed that there is significant correlation between the scores that the nurses got and the years of their experiences, that is mean we have to choose nurses having more years of experience to work in the PBU, that is to do a perfect job .

\section{Conclusions}

1- Most nurses who work in PBU are females, $50 \%$ of them aged between $30-50$, more than $50 \%$ are secondary schools graduates, $50 \%$ of them had more than 10 years of experience 2-only $3 \%$ had a participation in training sessions

3- they had a good level of knowledge were the mean of scores they get was about $74 \%$, all are save nurses because they all passed the evaluation (succeed )

4- Nurses in Basra hospital for Maternity and childhood and Amani general hospital had the highest scores i.e. they are having the best knowledge.

5- There is significant correlation between the scores that the nurses got and the years of their experiences.

\section{Recommendation}

1- We have to insist on the continuous education session for all nurses working at PBU, and all nurses have to share in such sessions

2- We have to open PBU at all Basra hospitals

3- We have to increase the graduation level to the PBU staff, to college level

\section{References}

[1] Whitfield, Jonathan M.; Peters, Beverly A.; Shoemaker, Craig (July 2004). "Conference summary: a celebration of a century of neonatal care". Proceedings (Dallas: Baylor University Medical Center) 17 (3): 255-258. Retrieved August 26, 2010.

[2] Harper, Douglas. "Neonatal". Online Etymology Dictionary. Douglas Harper. Retrieved October 26, 2010.

[3] "Frequently Asked Questions". Global Unity for Neonatal Nurses. Boston: Council of International Neonatal Nurses. 2009. Retrieved October 26, 2010.

[4] "Neonatal Nurse". Nurses for a Healthier Tomorrow. Nurses for a Healthier Tomorrow. Retrieved October 26, 2010.

[5] "Digital Bibliotheca - Michener Digitalisierungszentrum". Digitale-sammlungen.de.

[6] "Neonatology on the Web: Cardigan - An Essay upon Nursing 1749".neonatology.org.

[7] Glencoe H, Cousins S, Oestergaard M, teal. National, regional and worldwide estimates of preterm birth. The Lancet, June 2012. 9; 379(9832):2162-72. Estimates from 2010).

[8] Rodriguez RJ, Martin RJ, and Far-off, AA. Respiratory distress syndrome and its management. Far-off and Martin (eds.) 
Neonatal-perinatal medicine: Diseases of the fetus and infant; seventh ed. (2002): 1001-1011. St. Louis: Mosby.

[9] Pediatrics Vol. 114 No. 5 November 1, 2004 pp. 1341-1347.

[10] Neonatology.Org --> Equipment In The NICU Created 1/25/2002 / last modified 6/9/2002. Retrieved on September 2, 2009.

[11] Humidity control tool for neonatal incubator 1998: Abdi he M; Forges G; Deland S; Bach V; Villon P; Liberty J P, Medical \& biological engineering \& computing 1998; 36(2): 241-5.

[12] Toward Improving the Outcome of Pregnancy (1993).

[13] Singh, Maharani (2010). Care of the Newborn. pp. 4-5.

[14] Bliss website http://www.bliss.org.uk/different-levels-of-care

[15] Milligan DWA, Caruthers P, Mackey B, Ward Platt MP, Collingwood Y, Wooer L, Gibbons J, Draper E, Manktelow BN. 'Nursing Workload in UK tertiary neonatal units' in Archives of Disease in Childhood published online 30 Jun 2008.

[16] Dunn, P. M. (1995). "Professor Pierre Badin (1846-1907) of Paris, and modern perinatal care". Archives of disease in childhood. Fetal and neonatal edition 73 (3): F193-F195.

[17] Milligan DWA, Caruthers P, Mackey Betel. 'Nursing Workload in UK tertiary neonatal units' in Archives of Disease in Childhood published online 30 Jun 2008.

[18] Seakale L.T. Khoza1A.A. Tale. Knowledge, attitudes and practices of neonatal staff concerning neonatal pain management. The journal of democratic nurses of South Africa. Vole 37, No 2 (2014).
[19] Xanthine Cubbish, Jota Saran, Jeeves and Geetanjali. EFFECTIVENSS OF EDUCATIONAL INTERVENTION ON NEONATAL RESUSCITATION AMONG NURSING PERSONNEL, Health and Population - Perspectives and Issues 35(1), 36-45, 2012.

[20] Nasr in Khalesi, Fatimah Shoal Anjom, Golan Rezaeiezadeh, Zahra Farahani. Evaluation of Parents' Awareness and Knowledge about Problems and Issues Related to Their Premature Infants in an Iranian Hospital. Open Journal of Nursing, 2015, 5, 465-469.

[21] Alton M, Miracle J, Brandon D. One Intensive Care Nursery's Experience with Enhancing Patient Safety. Adv. Neonatal Care. 2006; 6(3): 112-9.

[22] Anita M, Snider L. The art of developmental care in the NICU: a concept analysis. J Adv. Knurs 2003; 41(3): 223-32.

[23] Laguna M. Priorities para el gobierno de la ciudad de Buenos Aires en la assistance neonatologist. Rev Hops Mat in Ramón Sadr. 2000; 120(3): 127-32.

[24] Hendricks-Munoz KD, Prendergast CC. Barriers to provision of developmental care in the neonatal intensive care unit: neonatal nursing perception. Am J Perinatal? 2007; 24(2): 71-7.

[25] Lei $\mathrm{n}^{\circ} 7.498$, de 25 de junco de 1986 (BR). Dispose sober a Regulamentação do Exercício da Enfermagem e ad outros providences. Dario Official da Union. Brasília (DF), de 26 de junco de 1986. Saco I - fly. 9.273 A 9.275. 